\title{
Structural analysis of human chromosomes by atomic force and light microscopy in relation to the distribution of topoisomerase IIa
}

\author{
Osamu Hoshi and Tatsuo Ushiki \\ Division of Microscopic Anatomy and Bio-imaging, Niigata University Graduate School of Medical and Dental Science, \\ Niigata, Japan
}

\begin{abstract}
Summary. The relationship between the higher-order structure of human metaphase chromosomes and the distribution of topoisomerase II $\alpha$ was analyzed by a comparison of atomic force microscope (AFM) and fluorescence microscope images of the same chromosome. AFM imaging of chromosomes in liquid revealed the presence of alternating ridges and grooves on the surfaces of the sister chromatids. In contrast, the fluorescence image of the chromosomes stained with the antitopoisomerase II $\alpha$ antibody showed that the fluorescence intensity of topoisomerase II $\alpha$ was not uniform and that there were alternating strong and weak spots along the chromosome axes. A comparison of the AFM image with a fluorescence microscope image of the same chromosome further demonstrated that ridges and grooves corresponded to strong and weak fluorescence intensities of topoisomerase II $\alpha$, respectively. These findings suggest that the distribution of topoisomerase II $\alpha$ has a close connection with the higher-order structure of human metaphase chromosomes.
\end{abstract}

Received February 4, 2009

Address for correspondence: Dr. Osamu Hoshi, Division of Microscopic Anatomy and Bio-Imaging, Niigata University Graduate School of Medical and Dental Sciences, Asahimachi-Dori 1, Chuoku, Niigata, 951-8150 Japan

Phone: +81-25-227-2062, Fax: +81-25-224-1767

E-mail: o-hoshi@med.niigata-u.ac.jp

\section{Introduction}

The higher-order structure of chromosomes has not yet been completely elucidated though various models have been proposed based on transmission electron microscope (TEM) studies (Dupraw, 1965; Bak et al., 1977, Marsden and Laemmli, 1979; Rattner and Lin, 1985). Because TEM images are two dimensional, scanning electron microscope (SEM) studies have been also performed to visualize directly the three-dimensional ultrastructure of chromosomes. However, the specimens should be fixed, dried and metal coated for this purpose, procedures often induce preparation artifacts on the the structure of specimens to be studied. As a means of overcoming these limitations, we have been investigating in atomic force microscopy (AFM) to clarify the higher-order structure of the chromosomes, especially in a liquid environment (Ushiki and Hoshi, 2008). These studies revealed that the chromatid arm of the human metaphase chromosome has ridges and grooves along its length and that these ridges and grooves roughly correspond to the G/Q-positive and G/Q-negative bands, respectively (Ushiki et al., 2002; Hoshi et al., 2007; Ushiki et al., 2008).

The chemical components of the chromosome have been also studied in relation to the structure of the chromosomes. Among them, topoisomerase II $\alpha$ is known as a scaffold protein of the chromosome as well as a catenation/decatenation enzyme. Immunolocalization studies revealed that topoisomerase II $\alpha$ is enriched in the chromosome axes throughout the entire chromosomal body (Earnshaw and Heck, 1985; Gasser et al., 1986; Boy de la Tour and Laemmli, 1988; Taagepera et al., 1993; Maeshima and Laemmi, 2003; Kirreva et al., 2004). In our previous study, we showed that the axial localization 
of topoisomerase II $a$ becomes much clearer in the prometaphase and metaphase than in the prometaphase of human chromosomes derived from lymphocytes (Hoshi et al., 2007a). Our findings also indicated that the fluorescence intensity of topoisomerase II $\alpha$ dose not appear to be uniform along the chromosome axes but is distributed in spots along the axes. The pattern of these intense spots in the chromosome arm seems to overlap with the banding pattern of G/Q-banded chromosomes. However, no comparison of the fluorescence intensity of topoisomerase II $\alpha$ and the surface structure of the chromatid arms of the same chromosome has been performed.

Although the relationship between the higherorder structure of chromosomes and the distribution of topoisomerase II $\alpha$ has been studied with immunoelectron microscopy (Maeshima et al., 2005), it is generally difficult to preserve both the antigenicity and the structural integrity of chromosomes by aldehyde fixation. On the other hand, in AFM imaging, the specimens need neither specific fixation nor metal coating (Ushiki, 2003; Hoshi et al., 2006), indicating that AFM of the chromosome has the advantage of obtaining high resolution images while preserving the antigenicity of the chromosome. The present study introduces a method for the direct comparison of atomic force microscopic images and immunofluorescence images of the same chromosome, which allows an analysis of the higher order structure of chromosomes in relation to the distribution of topoisomerase II $\alpha$.

\section{Materials and Methods}

\section{Spreads of human chromosomes}

Fresh heparinized peripheral blood from healthy donors was diluted 1:1 with Dulbecco's phosphate buffered saline (PBS). This was carefully layered on Ficoll-Paque Plus (Amersham Pharmacia Biotech, Uppsala, Sweeden) in a tube. Lymphocytes were collected from the interface layer after centrifugation at $550 \times \mathrm{g}$ for $30 \mathrm{~min}$. The isolated lymphocytes were cultured at $37^{\circ} \mathrm{C}$ under a $5 \%$ $\mathrm{CO}_{2}$ atmosphere for $72 \mathrm{~h}$ in a karyotyping medium (PBMAX, Gibco, California, USA). After $72 \mathrm{~h}$ incubation, the lymphocytes were arrested with $0.06 \mu \mathrm{g} / \mathrm{m} l$ colcemid (Demecolcine, Wako, Osaka) for $60 \mathrm{~min}$. The cell suspension was exposed to $0.075 \mathrm{M} \mathrm{KCl}$ for hypotonic treatment for $30 \mathrm{~min}$ and fixed with methanol-acetic acid (3:1). Spreads of metaphase chromosomes were made by dropping the cell suspension onto glass slides.

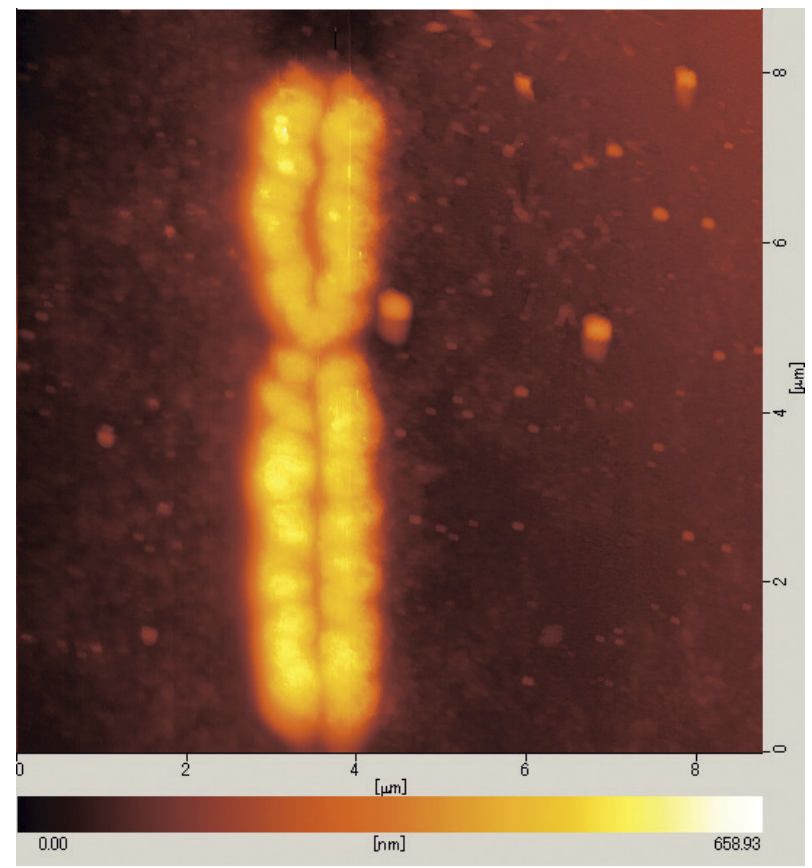

Fig. 1. AFM topographic image of a human chromosome (chromosome 2) in a PBS buffer solution. Alternating ridges and grooves are present on the surface of the chromatids. The arrangement of ridges and grooves corresponds to the paired sister chromatids.

\section{Atomic force microscopy}

The spreads of metaphase chromosomes were observed with a phase contrast microscope to determine the portions to be studied by AFM. After being photographed with a light microscope, the glass slides were cut into small pieces (about $1 \mathrm{~cm} \times 1 \mathrm{~cm}$ ), and the pieces bearing the chromosomes to be studied were selected and mounted on an open-type glass cell (about $2 \mathrm{~cm}$ in diameter and $5 \mathrm{~mm}$ in depth) with double-sided adhesive tape.

AFM observations were made with a commercial atomic force microscope (an SPA-400 microscope unit controlled by a SPI 4000 probe station, SII NanoTechnology, Chiba). For imaging chromosomes in liquid, the AFM was operated in a dynamic force mode (i.e., intermittent contact mode) in a phosphate buffered saline. The maximum scan range of the piezo scanner used in the present study was about $20 \mu \mathrm{m}$ in width $(\mathrm{x}-\mathrm{y})$ and $1.2 \mu \mathrm{m}$ in height $(\mathrm{z})$. The reduction in the oscillation amplitude was used as the feedback parameter by a slope detection technique, and commercially available V-shaped 

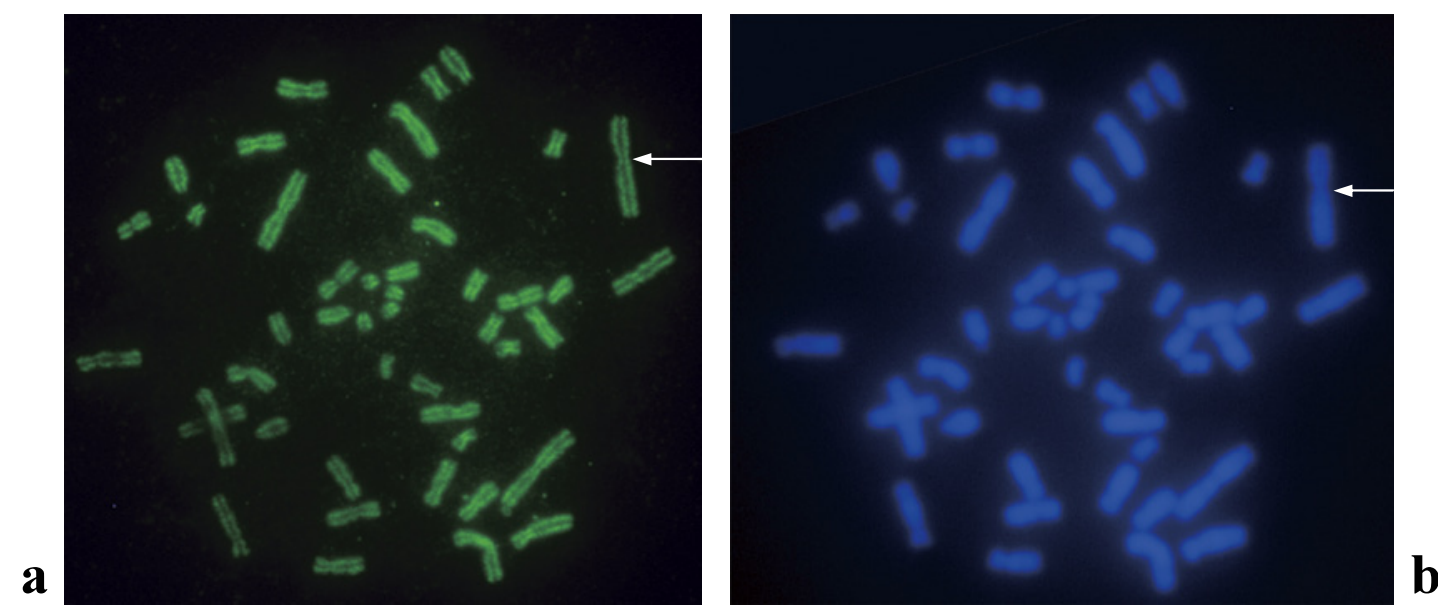

Fig. 2. Fluorescent image of human metaphase chromosomes stained with the anti-topoisomerase II $\alpha$ antibody (a). The fluorescence intensity is not uniform but slightly alternates between strong and weak spots. The specimen was counterstained with DAPI (b). Arrows indicate chromosome 2, which is shown by AFM in Figure 1.

silicon nitride cantilevers with a nominal spring constant of $0.32 \mathrm{~N} / \mathrm{m}$ (DNP-S20, Veeco Instruments Inc., New York, USA) were used for imaging in the liquid; the resonance frequency was normally about $12.5 \mathrm{kHz}$ in the liquid. The images obtained were composed of $512 \times 512$ pixels with height information and displayed in color gradations that represented the height difference.

\section{Immunostaining for chromosomes}

After the AFM images were obtained, the samples were stained with an anti-topoisomerase II $\alpha$ antibody as follows. After blocking with $10 \%$ fetal bovine serum (FBS) in $0.1 \%$ Triton X-PBS for $30 \mathrm{~min}$, the chromosomes were incubated with an anti-topoisomerase II $\alpha$ antibody (Calbiochem, USA) for $24 \mathrm{~h}$ and then with a fluorescence-labeled secondary antibody (Alexa Fluor 488 goat anti-mouse $\operatorname{IgG}[\mathrm{H}+\mathrm{L}]$; Molecular Probes, Oregon, USA) for $12 \mathrm{~h}$. Specimens were counterstained with $1 \mu \mathrm{g} / \mathrm{ml} \mathrm{4}$, 6-diamino-2-phenylindole dihydrochroride (DAPI). They were observed with a fluorescence microscope (ECLIPSE TE2000-U; Nikon, Tokyo) and photographed using a digital image acquisition system (Olympus; DP71, Tokyo).

\section{Results}

Before the AFM imaging of chromosomes, it was necessary to select suitable spreads of metaphase chromosomes without any overlap by observing samples with phase contrast microscopy. For AFM imaging, the interaction force between the tip and samples needed to be carefully adjusted because the chromosomes were easily deformed under inappropriate conditions. When the imaging conditions were suitable, the shape of chromosomes was clearly observed by AFM even in liquid. Thus, AFM imaging showed the presence of ridges and grooves on the surface of the sister chromatids (Fig.1). The arrangement of these ridges and grooves corresponded roughly to the paired sister chromatids. The height of the chromosomes ranged from 200 to $300 \mathrm{~nm}$.

After AFM imaging, the same specimens were immunostained with the topoisomerase II $\alpha$ antibody, followed by counterstaining with DAPI to identify the positive portion of topoisomerase II $\alpha$ in the whole chromosomes. Immunocytochemistry for topoisomerase II $\alpha$ thus showed that this enzyme was enriched in the chromosome axes along the length of the chromatids (Fig. 2a). The topoisomerase II $\alpha$ fluorescence appeared in spots along the chromosome axes, with intense fluorescence spots alternating with weak ones.

Comparison of AFM images with fluorescent images of the same chromosome also indicated that the ridged portions roughly showed intense topoisomerase II $\alpha$ fluorescence whereas the grooves showed weak topoisomerase II $\alpha$ fluorescence intensity (Fig3. a-d). 


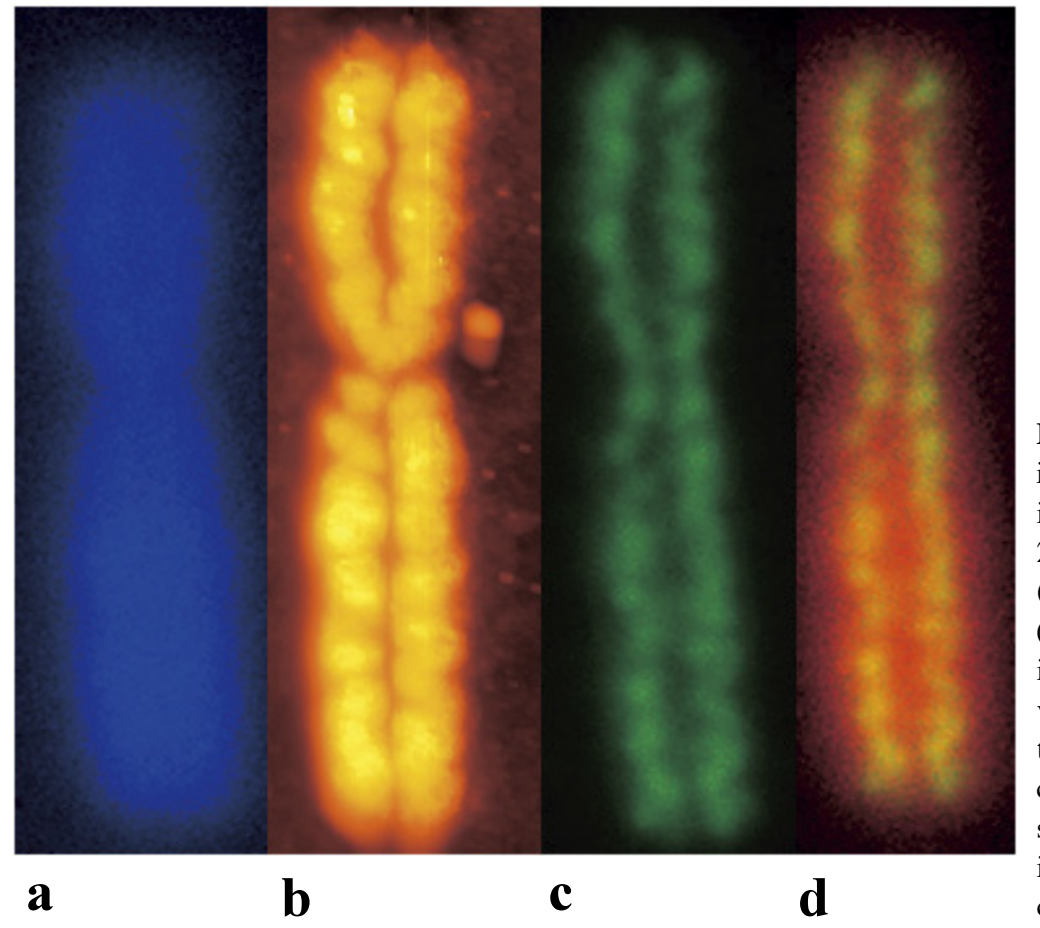

Fig. 3. Fluorescent (a, c, d) and AFM (b) images of the same chromosome. Fluorescent images $(\mathbf{a}, \mathbf{c})$ are closer views of chromosome 2 shown in Figure 2 (arrows) and AFM image (b) is the same as Figure 1. Superimposition (d) of the two images in a and c, is made in which blue is changed to red for a better visualization of the positive portion stained with the anti-topoisomerase II $\alpha$ antibody in the whole chromosome. Comparison between $\mathrm{b}$ and d clearly shows that the strong and weak fluorescence intensities of topoisomerase II $\alpha$ respectively correspond to ridges and grooves.

\section{Discussion}

The present AFM study has shown that the higher-order structure of the human metaphase chromosome is related to the distribution of topoisomerase II $\alpha$. Our previous studies revealed that AFM makes it possible to observe biological samples at high resolution without any special treatment (Ushiki, 2003; Hoshi et al., 2006). Thus, after AFM observation, the specimens can be immunostained to analyze the structure in relation to the distribution of proteins.

Topoisomerase II $\alpha$ is known to be important not only for the aggregation of chromatin fibers but also for their decatenation, indicating that the distribution of topoisomerase II $\alpha$ in the chromosome might be related to the higher-order structure of chromosomes. However, there are few reports focusing on this subject, except for a paper on the ultrastructural detection of topoisomerase II $\alpha$ using improved immunolabeling methods for electron microscopy (Maeshima et al., 2005).

As described above, we previously showed that the chromosomes are composed of a pair of the sister chromatids with ridges and grooves, and the compaction of the chromatin fibers appears stronger in the ridged regions than in the grooved regions (Ushiki et al.,
2002; Hoshi et al., 2007b; Ushiki et al., 2008). In the present study, we succeeded in obtaining AFM images and fluorescent images of topoisomerase II $\alpha$ in the same chromosome, and showed that the distribution of topoisomerase II $\alpha$ was denser in the ridged regions than in the grooved regions. Taken together into consideration, these findings indicate that the degree of compaction of the chromation fibers may be related to the amount of topoisomerase II $\alpha$. Our results also suggest that G-positive portions are strongly immunopositive for topoisomerase II $\alpha$ because the ridged portions roughly correspond to the G-positive portions of the chromosomes. Application of this method to other enzymes associated with chromosomal structures - such as condensin-will enable more comprehensive analyses of the higher-order structure of chromosomes.

In conclusion, we observed chromosomes by AFM in liquid, followed by immunofluorescent imaging for topoisomerase II $\alpha$ of the same chromosomes. A comparison of the topographic image with the distribution of topoisomerase II $\alpha$ in the same chromosome provides new information on the higher-order structure of the chromosomes. Correlation of AFM imaging with immunofluorescent imaging is indeed useful for analyzing the three-dimensional ultrastructure of the chromosome in relation to its chemical components. 


\section{Acknowledgements}

The authors would like to thank Mr. Y. Sano (Okayama University Graduate School of Medicine) for assistance with AFM imaging analysis.

\section{References}

Bak AL, Zeuthen J, Crick FHC: Higher-order structure of human mitotic chromosomes. Proc Nat Acad Sci USA 74: 1595-1599 (1977).

Boy de la Tour E, Laemmli UK: The metaphase scaffold is helically folded: sister chromatids have predominantly opposite helical handness. Cell 55: 937944 (1988).

Dupraw EJ: Macromolecular organization of nuclei and chromosomes: a folded fiber model based on wholemount electron microscopy. Nature 206:338-343 (1965).

Earnshaw WC, Heck MM: Localization of topoisomerase II in mitotic chromosomes. J Cell Biol 100: 1716-1725 (1985).

Gasser SM, Laroche T, Falquet J, Boy de la Tour E, Laemmli UK: Metaphase chromosome structure. Involvement of topoisomerase II. J Mol Biol 188: 613629 (1986).

Hoshi O, Ushiki T: Three-dimensional structure of G-banded human metaphase chromosomes observed by atomic force microscopy. Arch Histol Cytol 64: 475482 (2001).

Hoshi O, Owen R, Miles M, Ushiki T: Imaging of human metaphase chromosomes by atomic force microscopy in liquid. Cytogenet Genome Res 107: 28-31 (2004).

Hoshi O, Shigeno M, Ushiki T: Atomic force microscopy of native human metaphase chromosomes in a liquid. Arch Histol Cytol 69: 73-78 (2006).

Hoshi O, Hirota T, Kimura E, Komatsubara N, Ushiki T: Immunocytochemistry for analyzing chromosomes. In: Chromosome nanoscience and technology (Fukui K, Ushiki T, ed), CRC Press, Boca-Raton-London-New York, 2007a (p.81-91)
Hoshi O, Fukushi D, Ushiki T: Atomic force microscopy of human chromosomes in relation to their higherorder structure. In: Chromosome nanoscience and technology (Fukui K, Ushiki T, ed), CRC Press, BocaRaton-London-New York, 2007b (p.105-117)

Kireeva N, Lakonishok M, Kireev I, Hirano T, Belmont AS: Visualization of early chromosome condensation: a hierachical folding, axial glue model of chromosome structure. J Cell Biol 166:775-785 (2004).

Maeshima K, Eltsov E, Laemmli UK: Chromosome structure: improved immunolabeling for electron microscopy. Chromosoma 114: 365-375 (2005).

Marsden MPF, Laemmli UK: Metaphase chromosome structure: Evidence for a radial loop model. Cell 17: 849-858 (1979).

Maeshima K, Laemmli UK: A two-step scaffolding model for mitotic chromosome assembly. Dev Cell 4: 467-480 (2003).

Rattner JB, Lin CC: Radial loops and helical coils coexist in metaphase chromosomes. Cell 42: 291-295 (1985).

Taagepera S, Rao PN, Drake FH, Gorbsky GJ: DNA topoisomerase II alpha is the major chromosome protein recognized by the mitotic phosphoprotein antibody MPM-2. Proc Natl Acad Sci USA 90:84078411 (1993).

Ushiki T, Hoshi O, Iwai K, Kimura E, Shigeno M: The structure of human metaphase chromosomes: its histological perspective and new horizons by atomic force microscopy. Arch Histol Cytol 65: 377-90 (2002).

Ushiki T: Atomic force microscopy for imaging living organisms: from DNA to cell motion. In: Micromachines as tools for nanotechnology (Fujita $\mathrm{H}$ ed), Springer, Berlin-Heidelberg, 2003 (p. 121-130)

Ushiki T, Hoshi O: Atomic force microscopy for imaging human metaphase chromosome. Chromosome Res 16: 383-396 (2008). 